\title{
Numerical Solution of the Multiterm Time-Fractional Model for Heat Conductivity by Local Meshless Technique
}

\author{
Bander N. Almutairi, ${ }^{1}$ Ahmed E. Abouelregal $\left(\mathbb{D},{ }^{2,3}\right.$ Bandar Bin-Mohsin, ${ }^{1}$ M. D. Alsulami ${ }^{\circ},{ }^{4}$ \\ and Phatiphat Thounthong $\mathbb{C}^{5}$ \\ ${ }^{1}$ Department of Mathematics, College of Science, King Saud University, Riyadh 11451, Saudi Arabia \\ ${ }^{2}$ Department of Mathematics, College of Science and Arts, Jouf University, Al-Qurayyat, Saudi Arabia \\ ${ }^{3}$ Department of Mathematics, Faculty of Science, Mansoura University, Mansoura 35516, Egypt \\ ${ }^{4}$ University of Jeddah, College of Sciences and Arts at Alkamil, Department of Mathematics, Jeddah, Saudi Arabia \\ ${ }^{5}$ Renewable Energy Research Centre, Department of Teacher Training in Electrical Engineering, Faculty of Technical Education, \\ King Mongkut's University of Technology North Bangkok, 1518 Pracharat 1 Road, Bangsue, \\ Bangkok 10800, Thailand
}

Correspondence should be addressed to Ahmed E. Abouelregal; ahabogal@gmail.com

Received 24 March 2021; Revised 26 April 2021; Accepted 9 May 2021; Published 11 June 2021

Academic Editor: Nehad Ali Shah

Copyright (c) 2021 Bander N. Almutairi et al. This is an open access article distributed under the Creative Commons Attribution License, which permits unrestricted use, distribution, and reproduction in any medium, provided the original work is properly cited.

\begin{abstract}
Fractional partial differential equation models are frequently used to several physical phenomena. Despite the ability to express many complex phenomena in different disciplines, researchers have found that multiterm time-fractional PDEs improve the modeling accuracy for describing diffusion processes in contrast to the results of a single term. Nowadays, it attracts the attention of the active researchers. The aim of this work is concerned with the approximate numerical solutions of the three-term timefractional Sobolev model equation using computationally attractive and reliable technique, known as a local meshless method. Because of the meshless character and the simple application in higher dimensions, there is a growing interest in meshless techniques. To assess the reliability and accuracy of the proposed method, three test problems and two types of irregular domains are taken into account.
\end{abstract}

\section{Introduction}

In recent years, fractional partial differential equations (FPDEs) have drawn the consideration of numerous researchers to their applications in various fields of science and technology. Partial derivatives provide a flexible model and an extraordinary tool for description of capturing the history of the variable and genetic characteristics of various dynamic systems. Extensive research has been carried out in the advancement of numerical and analytical solutions of linear and nonlinear FPDEs [1-6]. However, several researchers have not succeeded in deriving and modeling many complex phenomena utilizing linear or nonlinear PDEs with integer order [7]. Subsequently, the fractional is taken as account and is a good solution to this problem [8]. In the current work, threeterm time-fractional Sobolev equation is considered which can be expressed as 


$$
\begin{aligned}
& \frac{\partial^{\beta_{1}} \mathscr{V}(y, z, t)}{\partial t^{\beta_{1}}}+\frac{\partial^{\beta_{2}} \mathscr{V}(y, z, t)}{\partial t^{\beta_{2}}}+\frac{\partial^{\beta_{3}} \mathscr{V}(y, z, t)}{\partial t^{\beta_{3}}}-\frac{\partial \nabla^{2} \mathscr{V}(y, z, t)}{\partial t}-\beta \nabla^{2} \mathscr{V}(y, z, t)+\gamma \nabla(\mathscr{V}(y, z, t) \nabla \mathscr{V}(\bar{z}, t)) \\
& +\delta \mathscr{V}(y, z, t)=F(\bar{z}, t), \quad(y, z) \in \Omega, 0<\beta_{3} \leq \beta_{2} \leq \beta_{1} \leq 1, t>0 .
\end{aligned}
$$

With the conditions,

$$
\begin{aligned}
& \mathscr{V}(y, z, 0)=\mathscr{V}_{0}(y, z), \\
& \mathscr{V}(y, z, t)=g_{1}(y, z, t), \quad(y, z) \in \partial \Omega,
\end{aligned}
$$

where $\nabla^{2}$ is the Laplacian and $\nabla$ denotes gradient operators, and $\beta, \gamma$, and $\delta$ the are known constants, whereas $\left(\partial^{\beta_{1}} / \partial t^{\beta_{1}}\right)$, $\left(\partial^{\beta_{2}} / \partial t^{\beta_{2}}\right)$, and $\left(\partial^{\beta_{3}} / \partial t^{\beta_{3}}\right)$ represent the Caputo derivative operator of order $0<\beta_{3} \leq \beta_{2} \leq \beta_{1} \leq 1$ for the function $\mathscr{V}(y, z, t)$.

In recent literature, various meshless methods have been utilized for the numerical solution of various PDE models almost in every discipline of science and engineering. In particular, the RBF-based meshless methods are the mainstream of these methods. The meshless nature is one of the main reasons behind the developing interest for such approaches. The meshless methods significantly reduce the complexity of dimensionality utilizing traditional methods such as the finite element and finite difference methods. Compared to mesh-based methods, these methods do not require mesh in the domain. The meshless methods have the ability to compute the solution in regular and irregular domain utilizing scattered or uniform nodes, which increases the priority and the advantages of meshless methods. As these facts show, these methods are really workable and useful numerical methods that can be applied to real-world challenging problems [9-17].

The RBF-based meshless methods have also some deficiencies like other numerical methods, in which the most important one is the dense ill-conditioned matrices and the selection of the optimal value of the shape parameter. To avoid these drawbacks, local meshless methods are the best alternatives, suggested by the researchers which are considered to be accurate and stable for the solution of diverse integer and fractional-order PDE models $[18,19]$. The local meshless methods are less sensitive to the change in shape parameters than the global version, and it produces wellconditioned sparse matrices. Furthermore, local version of meshless methods is considered to be more effective and efficient than global ones. In recent years, the abilities of various sorts of local meshless methods in different applications have been explored [20-22].

In the current research, we have implemented the local meshless method to approximate the numerical solution of three-term time-fractional model equation (1). For this purpose, multiquadric (MQ) radial basis functions (RBFs) are used. Furthermore, two types of irregular domains are also taken in numerical examples.

\section{Methodology of the Local Meshless Method}

According to the local meshless method, to approximate the derivatives of $\mathscr{V}(\overline{\mathbf{z}}, t)$ at the centers $\overline{\mathbf{z}}_{h}$ by the neighborhood of $\overline{\mathbf{z}}_{h}, \quad\left\{\overline{\mathbf{z}}_{h 1}, \overline{\mathbf{z}}_{h 2}, \overline{\mathbf{z}}_{h 3}, \ldots, \overline{\mathbf{z}}_{h n_{h}}\right\} \subset\left\{\overline{\mathbf{z}}_{1}, \overline{\mathbf{z}}_{2}, \ldots, \overline{\mathbf{z}}_{N^{n}}\right\}, n_{h} \ll N^{n}$, where $h=1,2, \ldots, N^{n}$, we have used $\overline{\mathbf{z}}=y$ and $\overline{\mathbf{z}}=(y, z)$ for one-dimensional and two-dimensional cases, respectively.

Now, considering the following case for onedimensional,

$$
\mathscr{V}^{(m)}\left(y_{h}\right) \approx \sum_{k=1}^{n_{h}} \lambda_{k}^{(m)} \mathscr{V}\left(y_{h k}\right), \quad h=1,2, \ldots, N
$$

Substituting the multiquadric $\operatorname{RBF} \psi\left(\left\|y-y_{p}\right\|\right)$ $=\sqrt{1+\left(c\left\|y_{h k}-y_{p}\right\|\right)^{2}}$ in (3),

$\psi^{(m)}\left(\left\|y_{h}-y_{p}\right\|\right)=\sum_{k=1}^{n_{h}} \lambda_{h k}^{(m)} \psi\left(\left\|y_{h k}-y_{p}\right\|\right), \quad p=h 1, h 2, \ldots, h n_{h}$.

Equation (4) in matrix form is

$$
\underbrace{\left[\begin{array}{c}
\psi_{h 1}^{(m)}\left(y_{h}\right) \\
\psi_{h 2}^{(m)}\left(y_{h}\right) \\
\vdots \\
\psi_{h n_{h}}^{(m)}\left(y_{h}\right)
\end{array}\right]}_{\psi_{n_{h}}^{(m)}}=\underbrace{\left[\begin{array}{cccc}
\psi_{h 1}\left(y_{h 1}\right) & \psi_{h 2}\left(y_{h 1}\right) & \cdots & \psi_{h n_{h}}\left(y_{h 1}\right) \\
\psi_{h 1}\left(y_{h 2}\right) & \psi_{h 2}\left(y_{h 2}\right) & \cdots & \psi_{h n_{h}}\left(y_{h 2}\right) \\
\vdots & \vdots & \ddots & \vdots \\
\psi_{h 1}\left(y h_{h_{h}}\right) & \psi_{h 2}\left(y_{h n_{h}}\right) & \cdots & \psi_{h n_{h}}\left(y h_{n_{h}}\right)
\end{array}\right]}_{A_{n_{h}}} \underbrace{\left[\begin{array}{c}
\lambda_{h 1}^{(m)} \\
\lambda_{h 2}^{(m)} \\
\vdots \\
\lambda_{h n_{h}}^{(m)}
\end{array}\right]}_{\lambda_{n_{h}}^{(m)}},
$$

where

$$
\psi_{p}\left(\left(y_{k}\right)\right)=\psi\left(\left\|y_{k}-y_{p}\right\|\right), \quad p=h 1, h 2, \ldots, h n_{h},
$$

for each $k=i 1, h 2, \ldots, h n_{h}$. Equation (5) in simple form is

$$
\boldsymbol{\psi}_{n_{h}}^{(m)}=\mathbf{A}_{n_{h}} \boldsymbol{\lambda}_{n_{h}}^{(m)}
$$


From (7), we obtain

$$
\boldsymbol{\lambda}_{n_{h}}^{(m)}=\mathbf{A}_{n_{h}}^{-1} \psi_{n_{h}}^{(m)}
$$

(3) and (8) implies

$$
\mathscr{V}^{(m)}\left(y_{h}\right)=\left(\boldsymbol{\lambda}_{n_{h}}^{(m)}\right)^{T} \mathbf{V}_{n_{h}}
$$

where

$$
\mathbf{V}_{n_{h}}=\left[\mathscr{V}\left(y_{h 1}\right), \mathscr{V}\left(y_{h 2}\right), \ldots, \mathscr{V}\left(y_{h n_{h}}\right)\right]^{T}
$$
as

$$
\begin{aligned}
& \mathscr{V}_{y}^{(m)}\left(y_{h}, z_{h}\right) \approx \sum_{k=1}^{n_{h}} \gamma_{k}^{(m)} \mathscr{V}\left(y_{h k}, z_{h k}\right), \quad h=1,2, \ldots, N^{2}, \\
& \mathscr{V}_{y}^{(m)}\left(y_{h}, z_{h}\right) \approx \sum_{k=1}^{n_{h}} \eta_{k}^{(m)} \mathscr{V}\left(y_{h k}, z_{h k}\right), \quad h=1,2, \ldots, N^{2} .
\end{aligned}
$$

For $\gamma_{k}^{(m)}$ and $\eta_{k}^{(m)}\left(k=1,2, \ldots, n_{h}\right)$, we continue as

$$
\begin{aligned}
& \boldsymbol{\gamma}_{n_{h}}^{(m)}=\mathbf{A}_{n_{h}}^{-1} \boldsymbol{\Phi}_{n_{h}}^{(m)}, \\
& \boldsymbol{\eta}_{n_{h}}^{(m)}=\mathbf{A}_{n_{h}}^{-1} \boldsymbol{\Phi}_{n_{h}}^{(m)} .
\end{aligned}
$$

The time derivative $\left(\partial^{\beta_{1}} \mathscr{V}(\overline{\mathbf{z}}, t) / \partial t^{\beta_{1}}\right)$ is discretized utilizing Caputo derivative [23], where $\beta_{1} \in(0,1)$ as

$$
\frac{\partial^{\beta_{1}} \mathscr{V}(\overline{\mathbf{z}}, t)}{\partial t^{\beta_{1}}}= \begin{cases}\frac{1}{\Gamma\left(1-\beta_{1}\right)} \int_{0}^{t} \frac{\partial \mathscr{V}(\overline{\mathbf{z}}, \vartheta)}{\partial \vartheta}(t-\vartheta)^{-\beta_{1}} \mathrm{~d} \vartheta, & 0<\beta_{1}<1, \\ \frac{\partial \mathscr{V}(\overline{\mathbf{z}}, t)}{\partial t}, & \beta_{1}=1 .\end{cases}
$$

Let $\tau$ be the time step size, and for the interval $[0, t]$, consider $t_{q}=q \tau, q=0,1,2, \ldots, Q$. We complete the timefractional derivative term as

$$
\begin{aligned}
\frac{\partial^{\beta_{1}} \mathscr{V}\left(\overline{\mathbf{z}}, t_{q+1}\right)}{\partial t^{\beta_{1}}} & =\frac{1}{\Gamma\left(1-\beta_{1}\right)} \int_{0}^{t_{q+1}} \frac{\partial \mathscr{V}(\overline{\mathbf{z}}, \vartheta)}{\partial \vartheta}\left(t_{q+1}-\vartheta\right)^{-\beta_{1}} \mathrm{~d} \vartheta, \\
& =\frac{1}{\Gamma\left(1-\beta_{1}\right)} \sum_{r=0}^{q} \int_{r \tau}^{(r+1) \tau} \frac{\partial \mathscr{V}(\overline{\mathbf{z}}, \vartheta)}{\partial \vartheta}\left(t_{r+1}-\vartheta\right)^{-\beta_{1}} \mathrm{~d} \vartheta, \\
& \approx \frac{1}{\Gamma\left(1-\beta_{1}\right)} \sum_{r=0}^{q} \int_{r \tau}^{(r+1) \tau} \frac{\partial \mathscr{V}\left(\overline{\mathbf{z}}, \vartheta_{r}\right)}{\partial \vartheta}\left(t_{r+1}-\vartheta\right)^{-\beta_{1}} \mathrm{~d} \vartheta .
\end{aligned}
$$

The term $\left(\partial \mathscr{V}\left(\overline{\mathbf{z}}, \vartheta_{r}\right) / \partial \vartheta\right)$ is approximated as follows:

$$
\frac{\partial \mathscr{V}\left(\overline{\mathbf{z}}, \vartheta_{r}\right)}{\partial \vartheta}=\frac{\mathscr{V}\left(\overline{\mathbf{z}}, \vartheta_{r+1}\right)-\mathscr{V}\left(\overline{\mathbf{z}}, \vartheta_{r}\right)}{\vartheta}+\mathcal{O}(\tau)
$$

Then, 


$$
\begin{aligned}
& \frac{\partial^{\beta_{1}} \mathscr{V}\left(\overline{\mathbf{z}}, t_{q+1}\right) \approx 1 / \Gamma\left(1-\beta_{1}\right) \sum_{r=0}^{q} \mathscr{V}\left(\overline{\mathbf{z}}, t_{r+1}\right)-\mathscr{V}\left(\overline{\mathbf{z}}, t_{r}\right) / \tau \int_{r \tau}^{(r+1) \tau}\left(t_{r+1}-\vartheta\right)^{-\beta_{1}} \mathrm{~d} \mathcal{V}}{\partial t^{\beta_{1}}}, \\
& =\frac{1}{\Gamma\left(1-\beta_{1}\right)} \sum_{r=0}^{q} \frac{\mathscr{V}\left(\overline{\mathbf{z}}, t_{q+1-r}\right)-\mathscr{V}\left(\overline{\mathbf{z}}, t_{q-r}\right)}{\tau} \int_{r \tau}^{(r+1) \tau}\left(t_{r+1}-\vartheta\right)^{-\beta_{1}} \mathrm{~d} \vartheta, \\
& = \begin{cases}\frac{\tau^{-\beta_{1}}}{\Gamma\left(2-\beta_{1}\right)}\left(\mathscr{V}^{q+1}-\mathscr{V}^{q}\right)+\frac{\tau^{-\beta_{1}}}{\Gamma\left(2-\beta_{1}\right)} \sum_{r=1}^{q}\left(\mathscr{V}^{q+1-r}-\mathscr{V}^{q-r}\right)\left[(r+1)^{1-\beta_{1}}-r^{1-\beta_{1}}\right], & q \geq 1, \\
\frac{\tau^{-\beta_{1}}}{\Gamma\left(2-\beta_{1}\right)}\left(\mathscr{V}^{1}-\mathscr{V}^{0}\right), & q=0 .\end{cases}
\end{aligned}
$$

Letting $\quad a_{0}=\left(\tau^{-\beta_{1}} / \Gamma\left(2-\beta_{1}\right)\right) \quad$ and $\quad b_{r}=(r+1)^{1-\beta_{1}}$

$-r^{1-\beta_{1}}, r=0,1, \ldots, q$, we have

$$
\frac{\partial^{\beta_{1}} \mathscr{V}\left(\overline{\mathbf{z}}, t_{q+1}\right)}{\partial t^{\beta_{1}}} \approx \begin{cases}a_{0}\left(\mathscr{V}^{q+1}-\mathscr{V}^{q}\right)+a_{0} \sum_{r=1}^{q} b_{r}\left(\mathscr{V}^{q+1-r}-\mathscr{V}^{q-r}\right), & q \geq 1 \\ a_{0}\left(\mathscr{V}^{1}-\mathscr{V}^{0}\right), & q=0 .\end{cases}
$$

The fractional derivative of order $\beta_{2}$ and $\beta_{3}$ can be found as above.

\section{Numerical Experiments}

This section examines the accuracy and applicability of the proposed method for the three-term time-fractional model (1). In the test problems, we have considered regular and irregular domains. This computation is considered to be regular and scattered nodes with regular and irregular domains. In this article, we have used the Crank-Nicholson scheme and multiquadric (MQ) RBF with shape parameter value $c=10$. Unless specifically stated, the spatial domain $[0,4]$ and time step size $\tau=0.002$ are used. Accuracy is measured as follows:

$$
\begin{gathered}
L_{\text {absolute }}=|\widehat{\mathbb{V}}-\mathscr{V}|, \\
\text { Max }- \text { error }=\max \left(L_{\text {absolute }}\right),
\end{gathered}
$$$$
\mathrm{RMS}=\sqrt{\frac{\sum_{h=1}^{N^{n}}\left(\widehat{\mathbb{V}}_{h}-\mathscr{V}_{h}\right)^{2}}{N}},
$$

where $\widehat{\mathbb{V}}$ is the exact solution, and $\mathscr{V}$ is the approximate solution.

Problem 1. Consider the model equation:

$$
\begin{aligned}
& \frac{\partial^{\beta_{1}} \mathscr{V}(y, z, t)}{\partial t^{\beta_{1}}}+\frac{\partial^{\beta_{2}} \mathscr{V}(y, z, t)}{\partial t^{\beta_{2}}}+\frac{\partial^{\beta_{3}} \mathscr{V}(y, z, t)}{\partial t^{\beta_{3}}}-\frac{\partial \nabla^{2} \mathscr{V}(y, z, t)}{\partial t}-\nabla^{2} \mathscr{V}(y, z, t)=F(y, z, t) \\
& 0<\beta_{3} \leq \beta_{2} \leq \beta_{1} \leq 1 \\
& t>0 .
\end{aligned}
$$

Having the exact solution,

$$
\mathscr{V}(y, z, t)=e^{-t} \sin (\pi y) \sin (\pi z), \quad(y, z) \in \Omega .
$$

The proposed meshless method is implemented for generating the required numerical results for Problem 1, which are given in Table 1 . Different values of a number of nodes $N$, fractional order $\beta_{1}=\beta_{2}=\beta_{3}$, and final time $t=1$ are used, whereas the error norms stand for max - error and RMS. These results revealed the fact that the recommended meshless method is capable of better results. Showing the accurate and efficient of the method, the results are compared with the exact solution for $\beta_{1}=\beta_{2}=\beta_{3}=0.1$, $\beta_{1}=\beta_{2}=\beta_{3}=0.3, \beta_{1}=\beta_{2}=\beta_{3}=0.5, t=1, t=2$, and for 
TABLe 1: Problem 1, approximate results for $t=1$.

\begin{tabular}{|c|c|c|c|c|c|c|}
\hline \multirow[b]{2}{*}{$N$} & \multicolumn{2}{|c|}{$\beta_{1}=\beta_{2}=\beta_{3}=0.2$} & \multicolumn{2}{|c|}{$\beta_{1}=\beta_{2}=\beta_{3}=0.5$} & \multicolumn{2}{|c|}{$\beta_{1}=\beta_{2}=\beta_{3}=0.8$} \\
\hline & Max - error & RMS & Max - error & RMS & Max - error & RMS \\
\hline $8^{2}$ & $8.5869 e-08$ & $3.9708 e-08$ & $3.8841 e-07$ & $1.2258 e-07$ & $4.9568 e-06$ & $1.7762 e-06$ \\
\hline $10^{2}$ & $8.2120 e-08$ & $3.9848 e-08$ & $4.8466 e-07$ & $1.4140 e-07$ & $5.8961 e-06$ & $1.9704 e-06$ \\
\hline $12^{2}$ & $8.8415 e-08$ & $3.9397 e-08$ & $5.2865 e-07$ & $1.6405 e-07$ & $6.1379 e-06$ & $2.1931 e-06$ \\
\hline
\end{tabular}

TABLe 2: Problem 1, approximate results using $N=8^{2}$ and $\beta=\beta_{1}=\beta_{2}=\beta_{3}$.

\begin{tabular}{|c|c|c|c|c|c|c|}
\hline \multicolumn{7}{|c|}{ Max - error } \\
\hline \multirow[b]{2}{*}{$\tau$} & \multicolumn{3}{|c|}{$t=1$} & \multicolumn{3}{|c|}{$t=2$} \\
\hline & $\beta=0.1$ & $\beta=0.3$ & $\beta=0.5$ & $\beta=0.1$ & $\beta=0.3$ & $\beta=0.5$ \\
\hline 0.2 & $1.1044 e-03$ & $9.9648 e-04$ & $7.9676 e-04$ & $7.9063 e-04$ & $7.1256 e-04$ & $5.7016 e-04$ \\
\hline 0.02 & $1.0790 e-05$ & $8.0577 e-06$ & $3.9095 e-06$ & $7.7071 e-06$ & $5.6947 e-06$ & $3.1952 e-06$ \\
\hline 0.002 & $1.0500 e-07$ & $4.3655 e-08$ & $3.8841 e-07$ & $7.4936 e-08$ & $3.0435 e-08$ & $2.9006 e-07$ \\
\hline
\end{tabular}

various values of time step size $\tau$. These results are computed using $N=8^{2}$ and are given in Table 2. One can observe from this table that only in few iterations, the suggested meshless method produced better results, and as the number of time iteration increases, the accuracy increase and the error norm reached up to $\max -$ error $\approx 10^{-10}$. As the condition number, stability, and accuracy of the RBF-based meshless methods heavily depend on the value of shape parameter $c$, a little change in shape parameter value causes instability and the results get diverge. But the suggested local meshless method is tested for Problem 1 in terms of condition number, stability, and accuracy as shown in Figure 1 for $N=10^{2}, \beta_{1}=\beta_{2}=\beta_{3}=0.5$, and $t=1$. This figure revealed that the suggested meshless method is stable, accurate, and given ideal low condition number $\approx 1$ for a long range of $c$ up to 2000. Figure 2 shows the absolute error using $\beta_{1}=\beta_{2}=$ $\beta_{3}=0.1$ and $\beta_{1}=\beta_{2}=\beta_{3}=0.8$ for $N=10^{2}$ and $t=1$. Better accuracy of the recommended algorithm can be seen in this figure.

Problem 2. Consider the model equation:

$$
\begin{aligned}
& \frac{\partial^{\beta_{1}} \mathscr{V}(y, z, t)}{\partial t^{\beta_{1}}}+\frac{\partial^{\beta_{2}} \mathscr{V}(y, z, t)}{\partial t^{\beta_{2}}}+\frac{\partial^{\beta_{3}} \mathscr{V}(y, z, t)}{\partial t^{\beta_{3}}}-\frac{\partial \nabla^{2} \mathscr{V}(y, z, t)}{\partial t}-\nabla^{2} \mathscr{V}(y, z, t)=F(y, z, t), \\
& 0<\beta_{3} \leq \beta_{2} \leq \beta_{1} \leq 1, \\
& t>0 .
\end{aligned}
$$

Having the exact solution,

$$
\mathscr{V}(y, z, t)=e^{y-z-t} \sin (\pi y) \sin (\pi z), \quad(y, z) \in \Omega .
$$

In Table 3, we have implemented the suggested algorithm for generating the numerical results for Problem 2 for $N=8^{2}, N=10^{2}, N=12^{2}, \beta_{1}=\beta_{2}=\beta_{3}=0.2, \beta_{1}=\beta_{2}=\beta_{3}$ $=0.4, \beta_{1}=\beta_{2}=\beta_{3}=0.4$, and $t=1, t=2$. The results are assessed in term of max - error and RMS. Accurate results have been obtained in this problem as well. Showing the applicability and efficacy of the propose method, the results are compared with the exact solution for various values of $\beta_{1}=\beta_{2}=\beta_{3} t$ and $\tau$ using $N=8^{2}$. These results are given in Table 4 . One can observe from this table that only in few iterations, the suggested meshless method produced better results, and as the number of time iteration increases, the accuracy increase and the error norm reached up to $\mathrm{RMS} \approx 10^{-9}$.

Just like the previous problem, the suggested method has been tested for Problem 2 in terms of condition number, stability, and accuracy as shown in Figure 3 for $N=10^{2}$, $\beta=0.5$, and $t=1$. It can easily be seen from the figure that the suggest meshless method is stable, accurate, and given ideal low condition number $\approx 1$ for a long range of $c$ up to 2000, whereas in Figure 4, we have shown a comparison of exact and approximate solutions for various values of time $t$ and brilliant match of both the solutions can be found in this figure.

One of the principle advantages of the meshless techniques over mesh-based techniques is the implementation in the irregular domain with ease. In this article, two types of challenging irregular domains are taken into account, which are displayed in Figure 5. In Table 5, we have shown the numerical results obtained by the suggested meshless method corresponding to the irregular domains for Problem 1 and Problem 2. We have considered the various value of $\beta$ 's, and the results are shown in form of $\max -$ error and RMS. It is observed from the table that better accuracy has been achieved in both domains. 


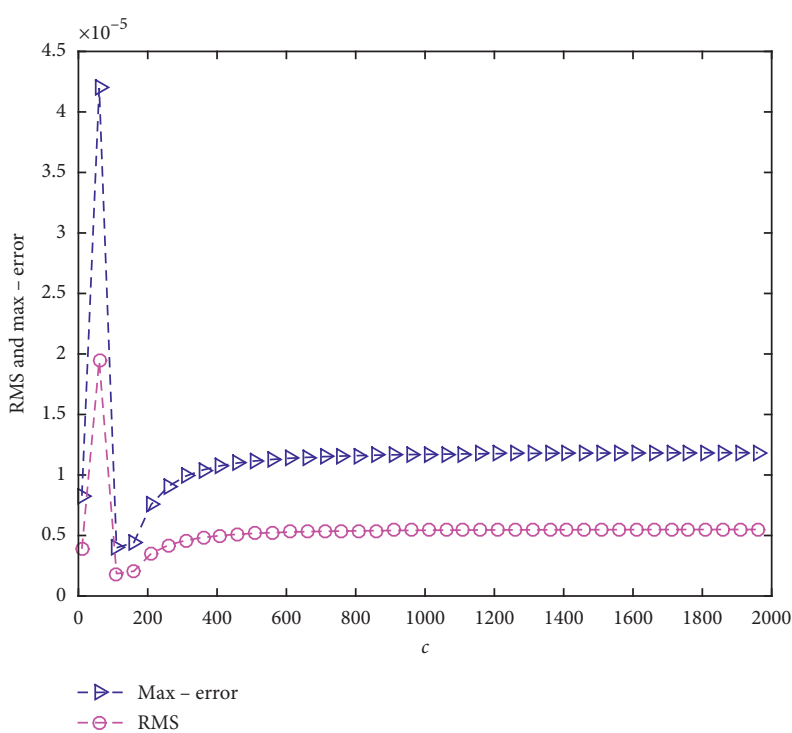

(a)

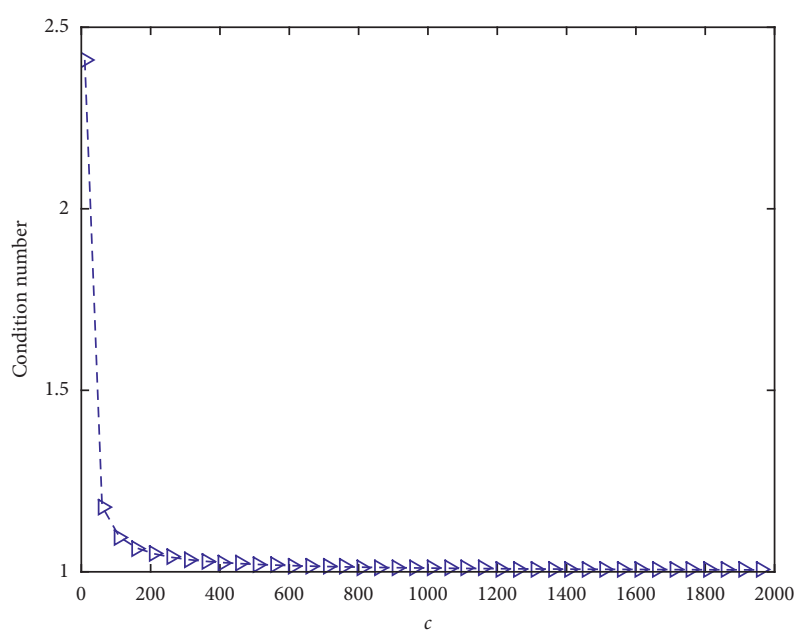

(b)

Figure 1: Problem 1. (a) $c$ and error norms, (b) $c$ and condition number.

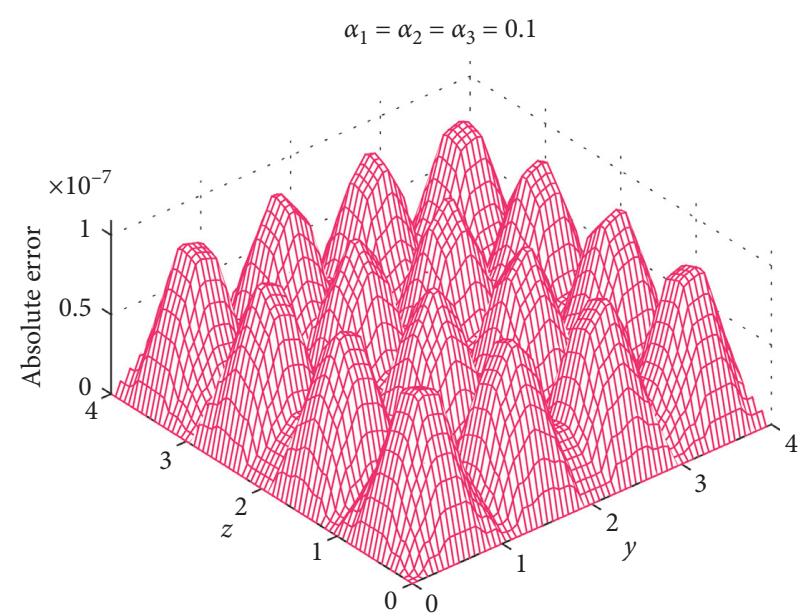

(a)

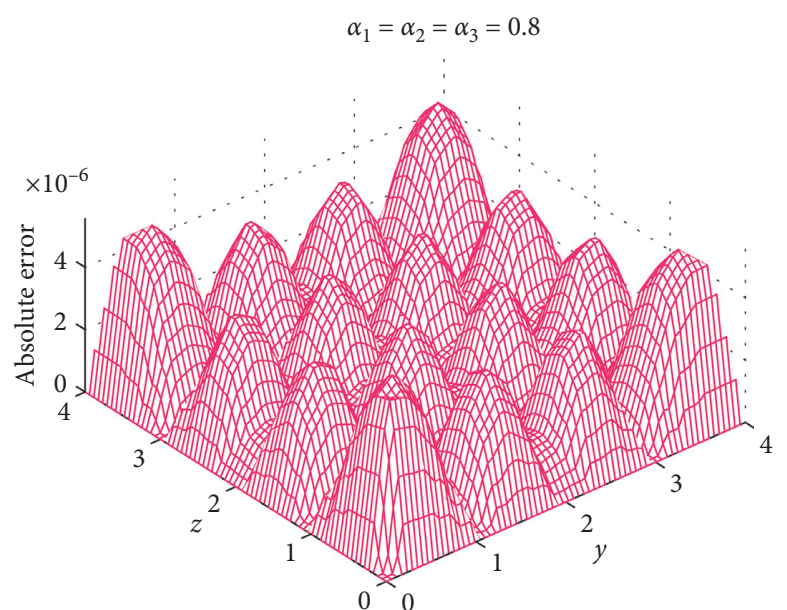

(b)

FIgure 2: Problem 1. Absolute error for $\beta=0.1$ (a) and $\beta=0.8$ (b), where $\beta=\beta_{1}=\beta_{2}=\beta_{3}$.

TABLE 3: Problem 2, approximate results for $t=1$.

\begin{tabular}{|c|c|c|c|c|c|c|}
\hline \multirow{2}{*}{$N$} & \multicolumn{2}{|c|}{$\beta_{1}=\beta_{2}=\beta_{3}=0.2$} & \multicolumn{2}{|c|}{$\beta_{1}=\beta_{2}=\beta_{3}=0.4$} & \multicolumn{2}{|c|}{$\beta_{1}=\beta_{2}=\beta_{3}=0.6$} \\
\hline & Max - error & RMS & Max - error & RMS & Max - error & RMS \\
\hline $8^{2}$ & $1.2325 e-06$ & $2.0404 e-07$ & $2.3271 e-06$ & $3.3074 e-07$ & $1.9346 e-05$ & $2.5361 e-06$ \\
\hline $10^{2}$ & $1.5895 e-06$ & $2.1784 e-07$ & $3.2329 e-06$ & $4.5136 e-07$ & $2.6251 e-05$ & $3.2570 e-06$ \\
\hline $12^{2}$ & $1.4030 e-06$ & $2.1123 e-07$ & $4.2754 e-06$ & $5.8259 e-07$ & $3.1278 e-05$ & $3.9590 e-06$ \\
\hline
\end{tabular}


TABle 4: Problem 2, approximate results for $N=8^{2}$.

\begin{tabular}{|c|c|c|c|c|c|c|}
\hline \multicolumn{7}{|c|}{ RMS } \\
\hline \multirow{2}{*}{$\tau$} & \multicolumn{3}{|c|}{$t=1$} & \multicolumn{3}{|c|}{$t=2$} \\
\hline & $\beta=0.2$ & $\beta=0.4$ & $\beta=0.6$ & $\beta=0.2$ & $\beta=0.4$ & $\beta=0.6$ \\
\hline 0.2 & $2.6139 e-03$ & $2.2018 e-03$ & $1.5260 e-03$ & $1.8624 e-03$ & $1.5697 e-03$ & $1.0945 e-03$ \\
\hline 0.02 & $2.3792 e-05$ & $1.2980 e-05$ & $4.3173 e-05$ & $1.6857 e-05$ & $9.2597 e-06$ & $3.3106 e-05$ \\
\hline 0.002 & $2.0404 e-07$ & $3.3074 e-07$ & $2.5361 e-06$ & $1.4425 e-07$ & $2.4742 e-07$ & $1.9042 e-06$ \\
\hline 0.0002 & $1.5812 e-09$ & $1.1891 e-08$ & $1.1014 e-07$ & $1.1152 e-09$ & $8.7439 e-09$ & $8.2401 e-08$ \\
\hline
\end{tabular}

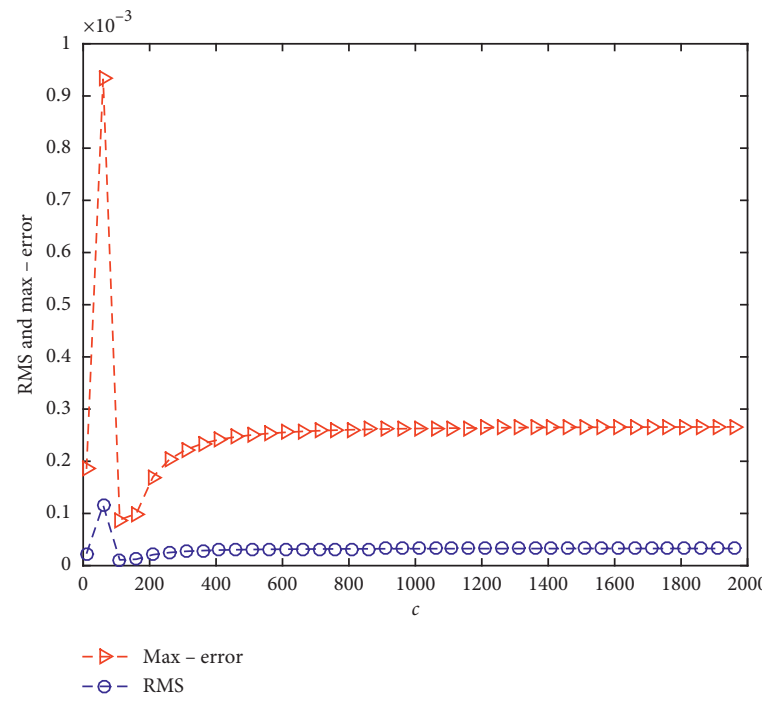

(a)

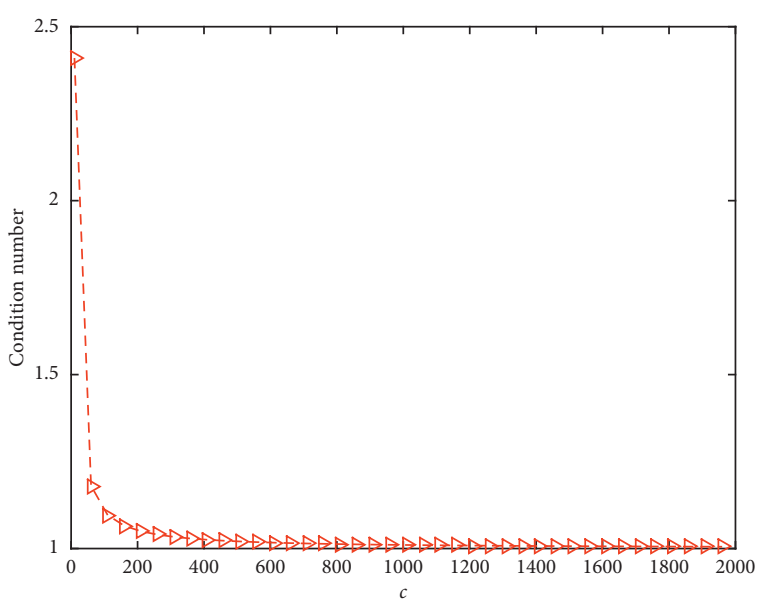

(b)

Figure 3: Problem 2. (a) $c$ and error norms. (b) $c$ and condition number.

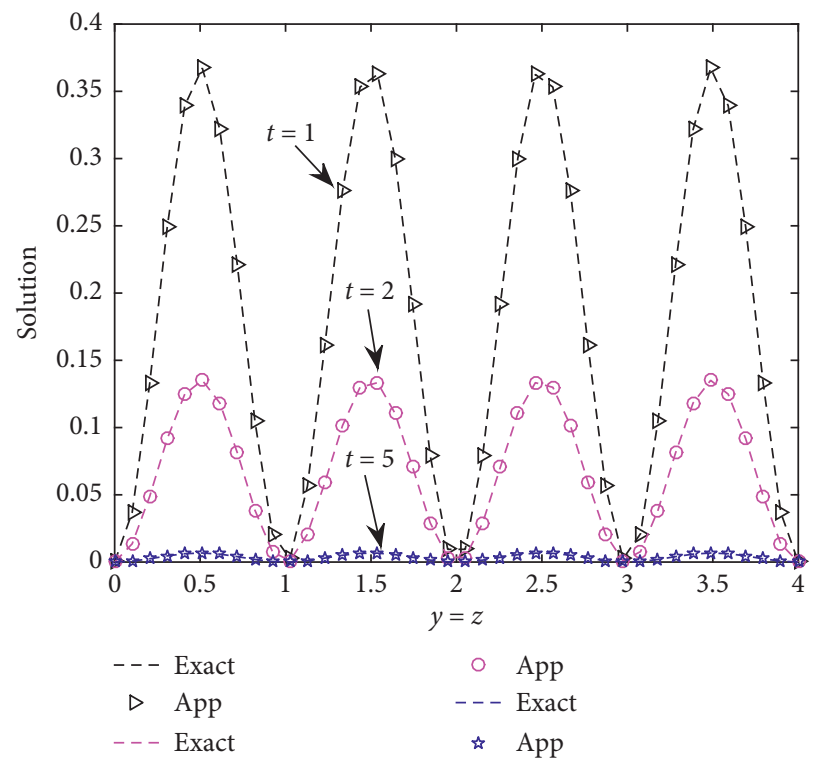

Figure 4: Problem 2, approximate and exact solution for indicated time. 


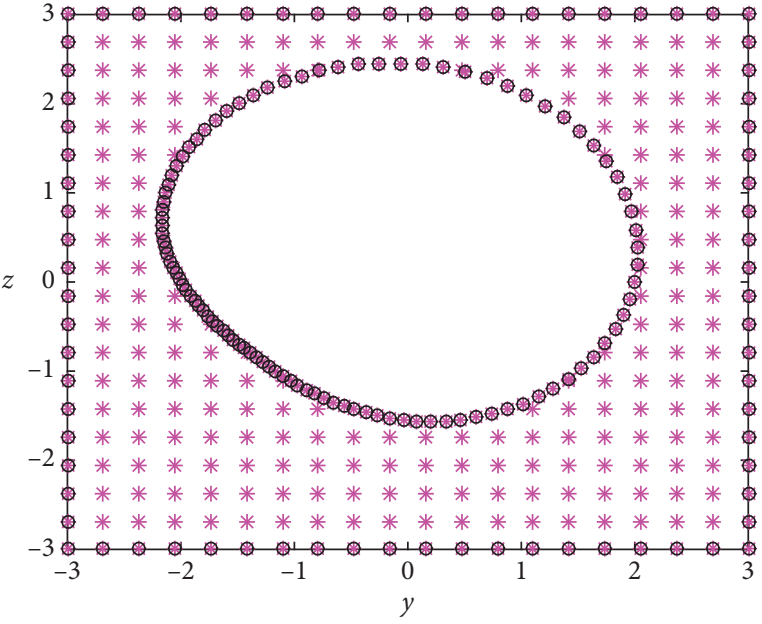

(a)

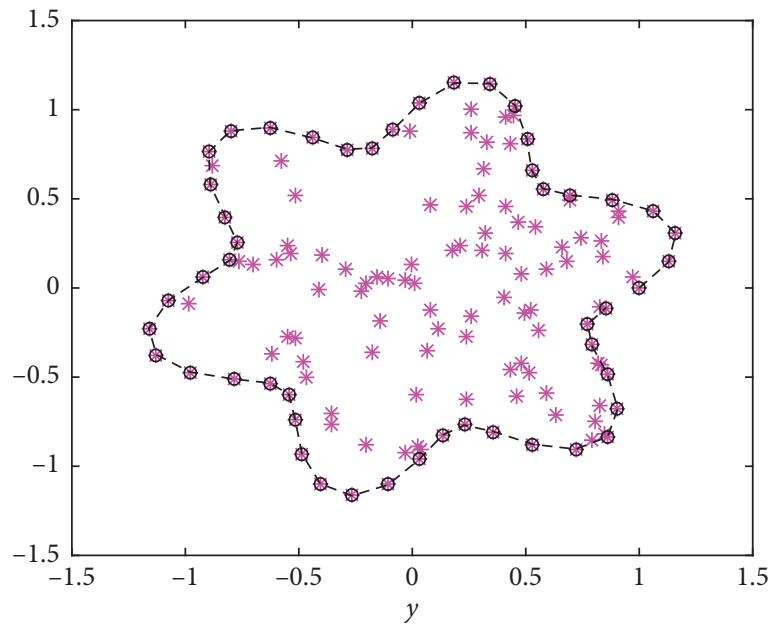

(b)

Figure 5: Computational domain 1 (a) and domain 2 (b).

TABLE 5: Approximate results corresponding to the irregular domains.

\begin{tabular}{lccccccc}
\hline & \multirow{2}{*}{ Domain } & \multicolumn{2}{c}{$\beta=0.2$} & \multicolumn{2}{c}{$\beta=0.5$} & \multicolumn{2}{c}{$\beta=0.8$} \\
& & Max - error & RMS & Max - error & RMS & Max - error & RMS \\
\hline \multirow{2}{*}{ Problem 1 } & Domain 1 & $2.8188 e-07$ & $7.0294 e-08$ & $3.2180 e-06$ & $8.2127 e-07$ & $3.3601 e-05$ & $9.0154 e-06$ \\
& Domain 2 & $1.9344 e-06$ & $3.4442 e-07$ & $2.0244 e-05$ & $3.8603 e-06$ & $1.1285 e-04$ & $2.6506 e-05$ \\
\hline \multirow{2}{*}{ Problem 2 } & Domain 1 & $9.0788 e-06$ & $1.1575 e-06$ & $9.5402 e-05$ & $1.0099 e-05$ & $1.0994 e-03$ & $1.1639 e-04$ \\
& Domain 2 & $3.3707 e-06$ & $6.0517 e-07$ & $3.2748 e-05$ & $6.3853 e-06$ & $1.2824 e-04$ & $3.2931 e-05$ \\
\hline
\end{tabular}

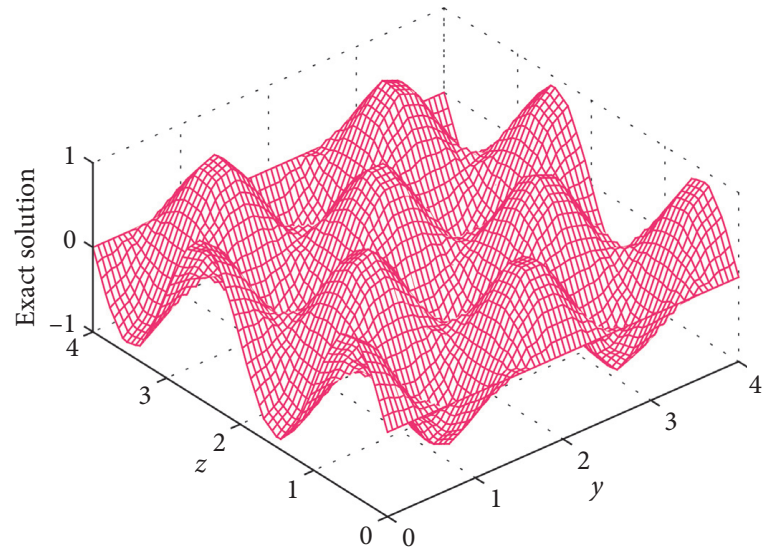

(a)

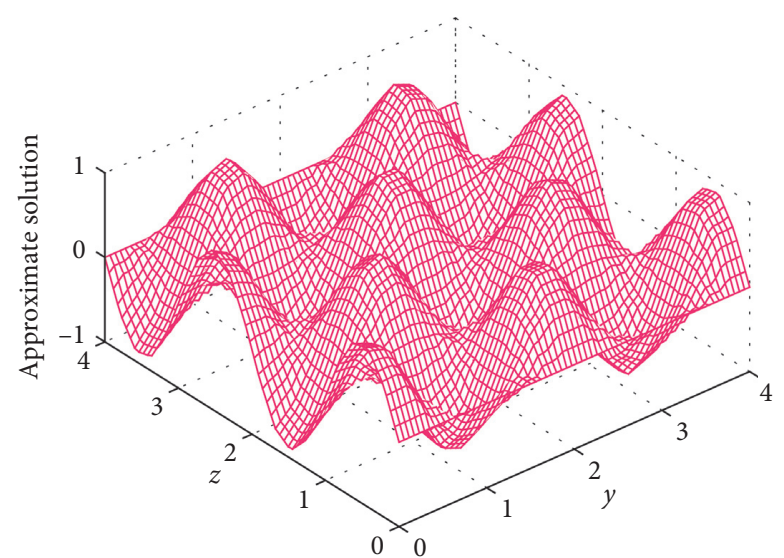

(b)

Figure 6: Problem 3. (a) Exact solution. (b) Numerical solution.

Problem 3. Consider the model equation:

$$
\begin{gathered}
\frac{\partial^{\beta_{1}} \mathscr{V}(y, z, t)}{\partial t^{\beta_{1}}}+\frac{\partial^{\beta_{2}} \mathscr{V}(y, z, t)}{\partial t^{\beta_{2}}}+\frac{\partial^{\beta_{3}} \mathscr{V}(y, z, t)}{\partial t^{\beta_{3}}}-\frac{\partial \nabla^{2} \mathscr{V}(y, z, t)}{\partial t}-\nabla^{2} \mathscr{V}(y, z, t)+\nabla(\mathscr{V}(y, z, t) \nabla \mathscr{V}(\overline{\mathbf{z}}, t)) \\
+\pi^{2} \mathscr{V}(y, z, t)=F(y, z, t), \quad(y, z) \in \Omega, 0<\beta_{3} \leq \beta_{2} \leq \beta_{1} \leq 1, t>0 .
\end{gathered}
$$




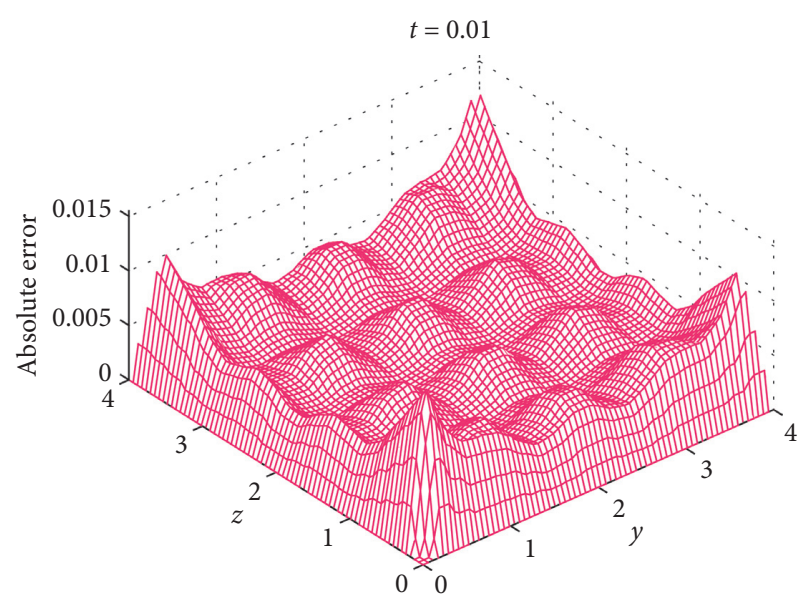

(a)

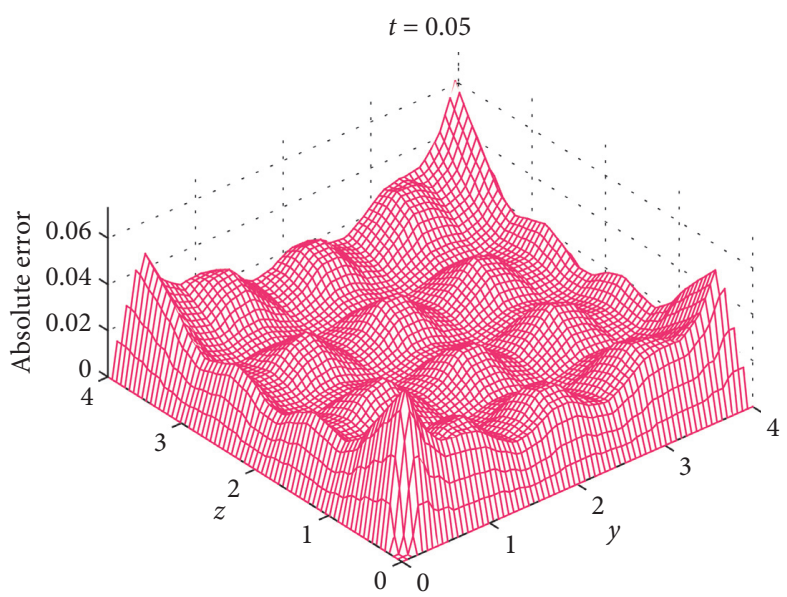

(b)

Figure 7: Problem 3, absolute error at (a) $t=0.01$ and (b) $t=0.05$.

Having the exact solution,

$$
\mathscr{V}(y, z, t)=e^{t} \sin (\pi y) \sin (\pi z), \quad(y, z) \in \Omega .
$$

In Figure 6, we have visualized the behavior of the exact and approximate solutions for the Problem 3 using $N=20^{2}$, $\beta_{1}=\beta_{2}=\beta_{3}=0.5$, and $t=0.1$, which show that the approximate solution is very compatible with the exact solution. In Figure 7, the absolute error is displayed for Problem 3.

\section{Conclusion}

In this study, our principle focused on the applicability and performance of the RBF-based local meshless method to approximate the numerical solution of three-term timefractional Sobolev equations. The computed results show that the proposed technique can take care of these sorts of problems amazingly and accurately. The local procedure leads to a sparse system of linear equations, and the solution is approximated with good accuracy. Three test problems are taken into account to test the effectiveness and accuracy of the proposed meshless method utilizing rectangular and two irregular domains. The numerical results demonstrate the high accuracy and effectiveness of the method. Given the current research, the proposed technique is a surprisingly powerful and successful tool for solving numerical problems of multiterm time-fractional PDEs found in various fields of science and technology.

\section{Data Availability}

The data that support the findings of this study are openly available at https://hindawi.com/publish-research.

\section{Conflicts of Interest}

The authors declare that they have no conflicts of interest.

\section{Acknowledgments}

The authors extend their appreciation to the Deanship of Scientific Research of King Saud University for funding this work through research group no. RG-1441-327.

\section{References}

[1] I. Ahmad, H. Ahmad, P. Thounthong, Y.-M. Chu, and C. Cesarano, "Solution of multi-term time-fractional PDE models arising in mathematical biology and physics by local meshless method," Symmetry, vol. 12, no. 7, p. 1195, 2020.

[2] N. A. Shah, I. Dassios, and J. D. Chung, "Numerical investigation of time-fractional equivalent width equations that describe hydromagnetic waves," Symmetry, vol. 13, no. 3, p. 418, 2021.

[3] H. Ahmad, T. A. Khan, I. Ahmad, P. S. Stanimirović, and Y.-M. Chu, "A new analyzing technique for nonlinear time fractional Cauchy reaction-diffusion model equations," Results in Physics, vol. 19, Article ID 103462, 2020.

[4] M. Inc, M. N. Khan, I. Ahmad, S.-W. Yao, H. Ahmad, and P. Thounthong, "Analysing time-fractional exotic options via efficient local meshless method," Results in Physics, vol. 19, Article ID 103385, 2020.

[5] N. A. Shah and J. D. Chung, "The analytical solution of fractional-order whitham-broer-kaup equations by an elzaki decomposition method," Numerical Methods for Partial Differential Equations, 2021.

[6] I. Ahmad, M. N. Khan, M. Inc, H. Ahmad, and K. S. Nisar, "Numerical simulation of simulate an anomalous solute transport model via local meshless method," Alexandria Engineering Journal, vol. 59, no. 4, pp. 2827-2838, 2020.

[7] S. T. R. Rizvi, I. Afzal, and K. Ali, "Chirped optical solitons for Triki-Biswas equation,” Modern Physics Letters B, vol. 33, no. 22, Article ID 1950264, 2019.

[8] R. Attia, D. Lu, and M. M. A. Khater, "Chaos and relativistic energy-momentum of the nonlinear time fractional duffing equation," Mathematical and Computational Applications, vol. 24, no. 1, p. 10, 2019.

[9] M. N. Khan, S. U. Islam, I. Hussain, I. Ahmad, and H. Ahmad, "A local meshless method for the numerical solution of space- 
dependent inverse heat problems," Mathematical Methods in the Applied Sciences, vol. 44, 2020.

[10] M. Nawaz, I. Ahmad, and H. Ahmad, "A radial basis function collocation method for space-dependent inverse heat problems," Journal of Applied and Computational Mechanics, vol. 6, 2020.

[11] P. Thounthong, M. Khan, I. Hussain, I. Ahmad, and P. Kumam, "Symmetric radial basis function method for simulation of elliptic partial differential equations," Mathematics, vol. 6, no. 12, p. 327, 2018.

[12] N. A. Shah, I. Dassios, E. R. El-Zahar, J. D. Chung, and S. Taherifar, "The variational iteration transform method for solving the time-fractional fornberg-whitham equation and comparison with decomposition transform method," Mathematics, vol. 9, no. 2, p. 141, 2021.

[13] H. Ahmad, A. R. Seadawy, and T. A. Khan, "Study on numerical solution of dispersive water wave phenomena by using a reliable modification of variational iteration algorithm," Mathematics and Computers in Simulation, vol. 177, 2020.

[14] H. Ahmad, A. R. Seadawy, T. A. Khan, and P. Thounthong, "Analytic approximate solutions for some nonlinear parabolic dynamical wave equations," Journal of Taibah University for Science, vol. 14, no. 1, pp. 346-358, 2020.

[15] N. A. Shah, E. R. El-Zahar, M. D. Aljoufi, and J. D. Chung, "An efficient approach for solution of fractional-order helmholtz equations," Advances in Difference Equations, vol. 2021, no. 1, pp. 1-15, 2021.

[16] Y.-M. Chu, N. A. Shah, P. Agarwal, and J. D. Chung, "Analysis of fractional multi-dimensional Navier-Stokes equation," Advances in Difference Equations, vol. 2021, no. 1, pp. 1-18, 2021.

[17] H. Ahmad, T. A. Khan, P. S. Stanimirovic, and I. Ahmad, "Modified variational iteration technique for the numerical solution of fifth order KdV type equations," Journal of Applied and Computational Mechanics, vol. 6, 2020.

[18] I. Ahmad, M. Ahsan, I. Hussain, P. Kumam, and W. Kumam, "Numerical simulation of PDEs by local meshless differential quadrature collocation method," Symmetry, vol. 11, no. 3, p. 394, 2019.

[19] I. Ahmad, M. Ahsan, Z.-U. Din, A. Masood, and P. Kumam, "An efficient local formulation for time-dependent PDEs," Mathematics, vol. 7, no. 3, p. 216, 2019.

[20] I. Ahmad, S.-U. Islam, and A. Q. M. Khaliq, "Local RBF method for multi-dimensional partial differential equations," Computers \& Mathematics with Applications, vol. 74, no. 2, pp. 292-324, 2017.

[21] C. Shu, Differential Quadrature and its Application in Engineering, Springer-Verlag, London, UK, 2000.

[22] I. Ahmad, H. Ahmad, A. E. Abouelregal, P. Thounthong, and M. Abdel-Aty, "Numerical study of integer-order hyperbolic telegraph model arising in physical and related sciences," The European Physical Journal Plus, vol. 135, no. 9, pp. 1-14, 2020.

[23] M. Caputo, "Linear models of dissipation whose Q is almost frequency independent--II," Geophysical Journal International, vol. 13, no. 5, pp. 529-539, 1967. 